\title{
Non-Player Character Personality and Social Connection Generation
}

\author{
Erica Jurado, Kirsten Emma Gillam, Joshua McCoy \\ Computer Science \\ University of California, Davis \\ Davis, CA USA \\ \{ejurado, kegillam, jamccoy\}@ucdavis.edu
}

\begin{abstract}
Increasing the behavioral nuance and interactivity of non-player characters in story worlds comes with a growing cost in the time and effort expended by authors. This paper proposes a system, Cast Affinity Satisfiability Toolkit (CAST), which uses answer set programming to lessen this burden while supporting author autonomy. CAST uses user-defined constraints about personality facets and interests to generate a cast of characters and their relationships. Once salient personality facets and character interests are defined, additional constraints may be supplied about character similarity and affinity. This refines not only character personality but the social connections between characters. Rules about affinity, or the attraction characters feel towards each other, may be used to weight personality facets and interests to generate consistent characters and their relationships that consider the significance of these traits. Although the quality of output is contingent upon the quality of constraints, CAST respects all author supplied constraints and provides the framework to generate NPCs that are consistent and whose relationships are tailored to reflect what is significant in the story world.
\end{abstract}

\section{CCS CONCEPTS}

- Logic programming and answer set programming • Design

\section{KEYWORDS}

Game design, interactive narrative, procedural content generation, character generation

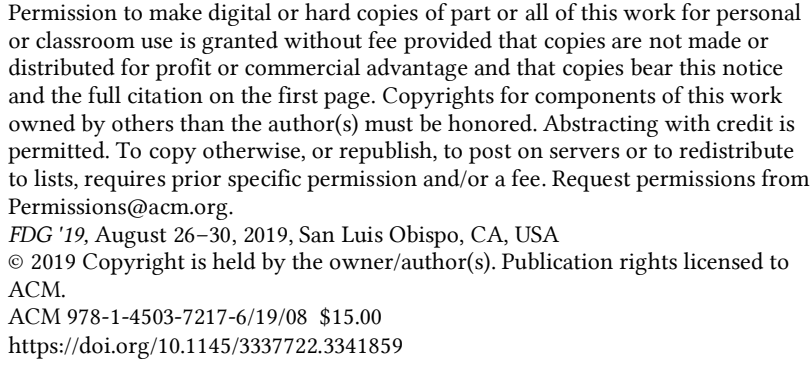
or classroom use is granted without fee provided that copies are not made or distributed for profit or commercial advantage and that copies bear this notice and the full citation on the first page. Copyrights for components of this work owned by others than the author(s) must be honored. Abstracting with credit is permitted. To copy otherwise, or republish, to post on servers or to redistribute to lists, requires prior specific permission and/or a fee. Request permissions from Permissions@acm.org.

FDG '19, August 26-30, 2019, San Luis Obispo, CA, USA

(c) 2019 Copyright is held by the owner/author(s). Publication rights licensed to ACM.

ACM 978-1-4503-7217-6/19/08 \$15.00

https://doi.org/10.1145/3337722.3341859

ACM Reference format:

Erica Jurado, Kirsten Emma Gillam and Joshua McCoy. 2019. Non-Player Character Personality and Social Connection Generation. In Proceedings of the 2019 Foundations of Digital Games Conference (FDG'19). FDG, San Luis Obispo, CA, USA, 7 pages. https://doi.org/10.1145/3337722.3341859

\section{Introduction}

Even in games that are exemplars of non-player character (NPC) design such as Star Wars: The Old Republic [11], Final Fantasy XV [13], or Bioshock Infinite [12], NPCs still lack the nuance and interactivity expected by players in many situations. This reduces the believability of the story world and limits the meaningfulness of player interaction. Hand crafting NPCs affords the author a high degree of control to try to create a rich social world in which player interaction is respected. However, with this authorial autonomy comes a high authorial burden. Procedural generation could aid in this process but defining characters in a succinct and meaningful way while respecting the author's choices is challenging [6]. Not only should the creator's autonomy be respected, but the resulting system of NPCs that is created should be coherent and provide an interesting space of play. Part of having an interesting space of play is having internally consistent characters with coherent relationships and social connections. In this paper we propose a system that uses answer set programming (ASP) and a rule set defining affinity to generate a cast of characters and their social connections.

Our system, Cast Affinity Satisfiability Toolkit (CAST), currently defines characters by their personality, interests, similarity, and affinity towards each other. The desired size of the set of characters must be defined and additional information about what personality traits authors deem important, interests they want their characters to have, and the affinity, or attraction, characters should have towards each other. While CAST supports arbitrary models of personality, it has an example rule set based on a subset of the Five Factor Model (FFM) traits. The FFM is a well-known taxonomy of personality that is both easily understandable and whose traits can be represented on a discrete numerical scale [2]. Although research about personality and the FFM does not provide concrete rules on how people with certain FFM values should interact with each other, the language used to describe the 


\section{NPC Personality \& Social Connection Generation}

facets provide a common vocabulary to describe personality. This enables users to describe their characters with a small set of words that is both intuitive to understand but powerful enough of a descriptor to create rules about it. Interests are vast and are story specific, so CAST does not provide suggestions for it. Instead the framework is provided to input interests appropriate for the world. If authors do not know all their interests, CAST can generate placeholders the decision of what interests characters should share can be deferred.

In addition to specifying these attributes characters have, CAST generates and determines the affinity characters have towards each other. Character similarity, or how close their facets and interests align, is an important metric, but similarity is not always a good indicator of the kind of relationships people might have. People who are very similar may be drawn to each other but common relationship tropes such as "opposites attract" may be encoded by defining affinity. To describe the extent to which people are drawn to each other, CAST uses rules about how personalities and interests interact to generate an affinity value. It is important to note that affinity is not compatibility. Compatibility has a positive connotation and is the extent to which people can harmoniously interact. People can be drawn to each other and constantly fight or be indifferent towards each other but still be able to interact without conflict. Affinity better captures this nuance and is thus used as a metric. The author may choose how to make use of this affinity metric in their game by drawing upon research on the FFM, media, common sense, and personal experience to create the appropriate familial, romantic, amical, and adversarial relationships between characters.

The cast and social network created by CAST can be used in multiple ways. This toolkit may be used as an iterative design tool to create a static set of characters for the game world. Alternatively, it may be used to repeatedly generate a new set of characters during runtime to create a new experience for the player each time they play. Regardless of the application, CAST procedurally generates characters that satisfy the author's constraints and choices to best aid in the NPC creation process.

\section{Related Work}

Content generation can be defined in terms of constraint satisfaction [5]. Answer set programming solvers can be used to find solutions that satisfy these constraints [10]. Past work in procedural content generation has used ASP to aid in maze and level generation, but comparatively little work has been done in using ASP to procedurally generate characters. Part of this is due to a common challenge in procedural content generation (PCG) that necessitates we must know what our generated content should look like as well as the process needed to generate it [8]. CAST draws upon past research done in PCG as well as on the FFM to help define what generated content should look like. PCG works to generate instances of content that fit given constraints. To do so both the generative process and the artifacts to be generated must be defined. However, what is desirable may

\section{FDG'19, August 26-30, 2019, San Luis Obispo, CA}

be unclear until something has been generated [10]. PCG inherently has a requirement of "ill-definedness" that can be resolved through iterative design [10]. CAST can be used in this way by offering the author a candidate solution to help better understand the traits they want their solution to have and reform constraints accordingly.

Procedural content generation has been used to aid in character creation, but it has been focused on the creation of physical attributes. Ontogenetic approaches have been used to alter a base character with different modular body parts to do this, but little work has been done to generate non-physical attributes [9]. Generating non-physical attributes such as personality provides different design constraints such as the encoding of soft attributes rather than physical traits. CAST draws inspiration from social psychology to discretize personality to define the artifacts that should be created.

Although there are different theories of personality, CAST's example rules are formed around the Five Factor Model (FFM), also known as the Big Five trait taxonomy or OCEAN personality model [2]. The underlying goal of this taxonomy was to define the overarching domain so instances within it could be easily understood and researchers could study domains of related personality characteristics [2]. The Big Five domains are extraversion, agreeableness, conscientiousness, neuroticism, and openness. The Big Five emerged from multiple studies from different labs and thus the specific variables researchers included are different [1]. Although factors of facets may vary across studies because of this, the fact that the labels differ does not mean the factors themselves are different [2]. Taxonomies such as the Big Five are fuzzy but, when using exemplars of each facet, are useful and descriptive.

The Big Five is defined with prototypical traits that are found across studies and integrating these interpretations of factors helps map the Big Five into common language. Additionally, when comparing Trait Descriptive Adjectives (TDA), NeuroticismExtraversion-Openness Five-Factor Inventory (NEO-FFI), and Big Five Inventory (BFI), there is very high convergent validity and a high level of internal consistency [2]. BFI and TDA most strongly converged and their mean discriminant correlations are low [2]. The lack of intercorrelations combined with high convergent validity among these Big Five models are a strength both within psychological studies and are a strength for authoring content based on it.

However, like any model, the Big Five has limitations. The Big Five does not provide a complete theory of personality but instead offers a model to account for relations among personality traits [7]. It cannot account for all variation in human personality, but this is inherently a problem with any hierarchical representation because abstraction results in information loss. The existence of this taxonomy, although broad and imperfect, still allows for the 


\section{Author Constraints: $\quad$ Cast Affinity Satisfiability Toolkit:}

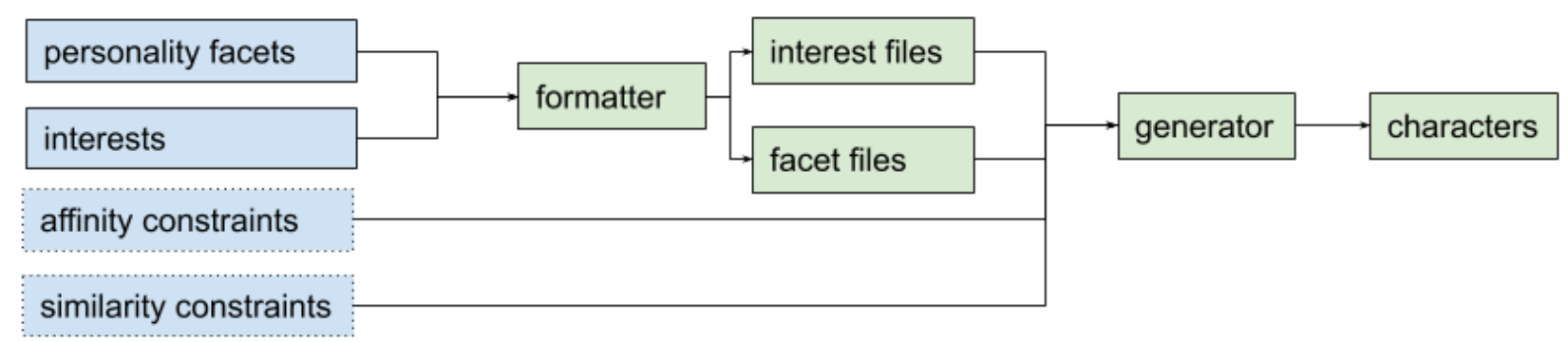

Figure 1: Personality facets, interests, affinity constraints, and similarity constraints are created by the author as outlined in section 3.1. Personality facets and interests are reformatted and the corresponding Clingo interest and facet files are generated. These files are used by the generator to create a cast of characters. Section 3.2 describes this generation process as well as the formation and significance of affinity and similarity constraints.

comparison and integration of dimensions of personality that might otherwise be disparate.

There are few systems utilizing the Big Five to model character behavior, but some work has been done creating crowd variation with the Big Five model. For example, the HiDAC (HighDensity Autonomous Crowds) system was extended to use the OCEAN model as a basis for agent personality [1]. Nominal behaviors were associated with these traits. Through specifying personality for an agent and tuning salient parameters within the model, HiDAC was then able to create interesting emergent behaviors [1]. CAST enables the author to specify and tune personality and interests as well as affinity rules. Emergent properties such as cliques may emerge but actualizing these properties into interesting behaviors still falls to the author when using CAST's output in their game.

\section{Tech Description}

CAST uses answer set programming (ASP) to determine the values of personality facets and interests as well as the similarity and affinity between characters. As shown in figure 1, CAST takes declarative statements about what the cast should look like such as the number of characters, facets or interests one or many characters should have, what attributes they should share with others, and the extent to which characters are similar or drawn to each other. CAST has a set of example affinity rules based on FFM relationship compatibility, common sense knowledge, and media for storytellers to use as a foundation for using CAST for their works.

\subsection{Author Constraints}

CAST has built-in constraints and definitions of what character affinities are desirable and the possible values of facets. The extent of similarities and affinities between characters and what is desirable is highly dependent on the author's goals and the world the generated characters will inhabit. For example, in a dramatic murder mystery, it may be desirable for characters to have more extreme traits and more varied affinities to accommodate a complex web of individuals with varying motives. In other contexts, such extreme characteristics and relationships can cause breaches in believability. In general, character diversity is desirable to create an interesting cast of heterogeneous NPCs but not so much diversity that relationships are volatile or characters too exaggerated. To fine-tune the kinds of characters, similarities, affinities, and relationships the world should have, additional constraints about these facts may be given.

In addition to supplying the exact number of characters to generate, authors can provide specific facet or interest values. They may specify any facet they wish, but the facets in the example set of affinity rules are formed around a subset of traits from the revised NEO taxonomy.

Character pairs may be specified to have a similarity value for a specific facet or interest. Additionally, constraints can be set for characters to have an overall similarity value. This is created by aggregating the similarity level of each facet and interest level. The pair similarity is increased by one for each high similarity or decreased by one for each low similarity and kept the same for neutral similarity.

However, this measure alone does not provide sufficient specificity and control for the author. The level of similarity between characters in general or for a specific facet can be given. For example, they may request that two characters have low similarity in compliance. Additionally, the author can specify that there be exactly, greater than, or less than $\mathrm{N}$ pairs of characters who have a pair similarity exactly, greater than, or less than $\mathrm{S}$.

These patterns serve to describe the overall set of characters and their relationships. Instead of having to define constraints between specific characters, this pattern ensures that a general description of arbitrary characters can be considered. More broadly, this empowers the user who has an idea about the overarching social state of the world they want to describe it accordingly. 


\section{NPC Personality \& Social Connection Generation}

For example, if the author knew they wanted a protagonist, Mario, and an antagonist, Bowser, with 6 supporting characters they would request the following:

character (mario).

character (bowser).

character $(1 \ldots 6)$.

If they know what they want personality facets or interests to be they can set it directly. By default personality and interests must be an integer between 1 and 10. This range balances the space searched by ASP and expressiveness. Supporting characters may also have specific values set by using their placeholder identifier. In this world our protagonist should be a little boastful, relatively assertive, and somewhat impulsive. In this story kidnapping princesses should be an interest that causes conflict. To do this the hero and protagonist's values for kidnapping princesses are drastically different. The other interest in this world is plumbing, and our hero should enjoy it and one of the supporting characters should have a bit of interest in it to ensure Mario has someone to relate to. These constraints would be written as follows:

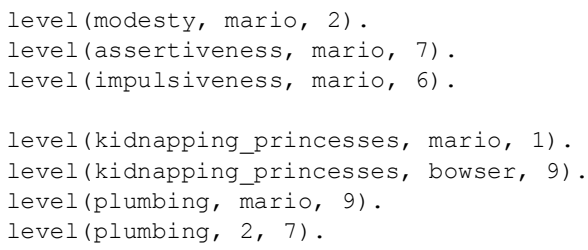

In addition to setting attributes for a character, CAST allows for constraints about similarity across pairs or groups of characters. Pair facet similarity must be less than or equal to the number of personality facets defined. In this world the personality traits that exist are modesty, assertiveness, and impulsiveness so the pair facet similarity must be a value between -3 and 3 . Negative values mean a trait is highly dissimilar where positive values mean a trait is very similar. For example, to make 2 out of 3 of the personality traits of Mario and Bowser dramatically different the author would need to make the following constraint:

pair_facet_similarity(mario, bowser, -2).

CAST also takes constraints about non-specific characters. To request that exactly 4 characters have a slight interest in kidnapping princesses and 4 characters are disinterested in kidnapping princesses the following constraint could be used:

match_n_attribute_level_min (4, kidnapping_princesses, 7). match_n_attribute_level_max (4, kidnapping_princesses, 4).

The cast of characters is 8 people so this constraint will encourage two distinct groups of people, one that enjoys kidnapping princesses and the other that does not.

When writing constraints, it is essential to remember the tradeoff between increasing specificity and satisfiability. More constraints

\section{FDG'19, August 26-30, 2019, San Luis Obispo, CA}

result in a space that more closely fits the author's vision, but if it is over specified there may not exist a space to meet those constraints. As such, iteratively and incrementally adding constraints will best help the author find the cast of characters they want. To see the full list of supported constraints, please see CAST's GitHub repository: https://github.com/EricaJurado/CastAffinity-Satisfiability-Toolkit.

\subsection{Generator}

The CAST generator is built in Clingo, an ASP system that grounds with gringo and solves with clasp [4]. In ASP it is possible to express a problem as a series of logical models $t$ provide solutions to the problem [4]. Gringo translates the provided logic programs with variables into the corresponding propositional logic program. Clasp solves the answer sets of the propositional logic program. Clingo combines these two tools. Clingo is, therefore, able to do ASP solving and reasoning over modules and is also able to handle linear constraints over integers [3]. Regardless of the order rules are written in the solution is the same if the constraints are the same. Additionally, it is guaranteed to terminate. These functionalities enable CAST to generate an answer set of possible characters and their attributes that meet the user's constraints, use integrity constraints to determine similarity, and always terminate regardless of satisfiability.

Each factor is defined by a series of facts and variables which is autogenerated by our file facet generator within CAST. Each supplied factor is defined as a facet. The factor itself may take a value between 1 and 10. Each character is paired with a value for that facet. Additionally, the extent to which a character is like another in that one facet is defined here. Similarity between two characters for a given facet may be high, neutral, or low meaning that the differences between their facet value is either within 2 , between 2 and 4, or more than 4, respectively.

CAST uses these facet definitions in combination with rules about similarity and affinity to generate the answer set. These may be supplied by the user to describe the similarity of each factor between arbitrary characters, specific values for facets, and constraints on the aggregate similarity between characters. However, another benefit CAST offers is an example set of affinity rules. Similarity alone may be insufficient to generate a compelling cast of characters. Similarity encompasses likeness across dimensions but does not necessarily correspond to how characters feel about each other or how characters are drawn to each other. This is necessary because feelings are not always reciprocated and the differences in affinity impact the existence of relationships and the nature of characters' interactions and behaviors. Differences in affinity also provide further ground for narrative possibilities as conflicts may arise when characters feel differently about each other.

Affinity rules may be written about personality facets or about interests. In the story world outlined in the previous section, conflict between characters should arise over the kidnapping of 


\section{NPC Personality \& Social Connection Generation}

princesses. The similarity metric would be useful in identifying which characters have strong differences in their interest in kidnapping princesses. Without additional affinity rules differences in interest level of kidnapping princesses have the same social weight as differences in interest of plumbing or differences in modesty, assertiveness, or impulsiveness. Kidnapping should be a polarizing interest and to correct for this the author would need to write affinity rules capturing this.

Characters who like kidnapping princesses should be drawn to other villainous characters who also like kidnapping princesses. Heroes opposed to the practice should be drawn to each other over their common disdain. Perhaps even more important to the conflict is that people who have strong but opposing feelings about kidnapping princesses should be repulsed by each other. Kidnappers likely feel some slight disdain for people who do not share that interest whereas heroes should hate those who enjoy kidnapping. To encode these the following affinity rules could be added to CAST:

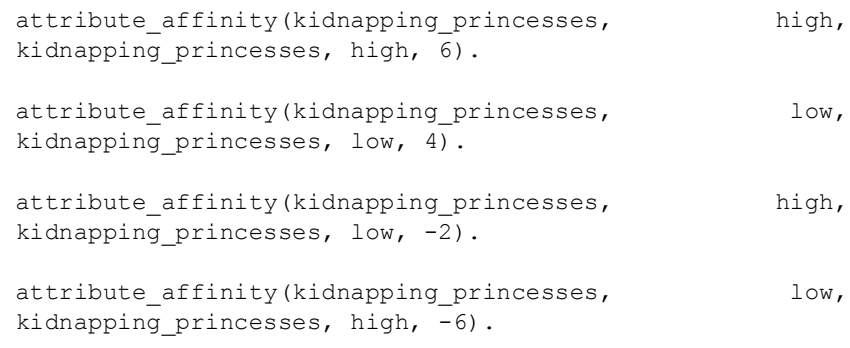

CAST provides the framework to describe characters in terms of personality, interests, and the dynamics between characters. With the inclusion of authored affinity rules, CAST can generate a cast of characters whose relationships are consistent with the world and with their identity.

\section{Evaluation}

Although CAST provides a way to iteratively generate a cast of characters that satisfies their constraints, it is important to note that this does not guarantee the quality of the output. If there exists a solution that satisfies the given constraints, CAST will find it. The example shown in this paper was selected to demonstrate the properties a generated cast may have, but not all solutions will demonstrate this. Under-defined requests may have a large answer set where most solutions provide little ground for narrative possibilities. Ill-defined requests that are satisfiable will yield an answer set but may not adhere to the author's vision. Properly defined requests enable CAST to generate a cast of characters with the desired similarity and affinity values to create desirable emergent properties that are consistent with the author's narrative. Ultimately CAST's biggest strength and weakness is quality of the output is contingent upon the user's vision and their ability to define requests to realize their vision. Additionally, even if the generated cast has internally consistent
FDG'19, August 26-30, 2019, San Luis Obispo, CA

characters with interesting social connections, authors must still decide how to make use of this cast.

One of the emergent properties that may occur when using CAST is cliques. In the example outlined in the previous section, the cliques seen in figure 2 are formed. Through constraining the similarities between pairs of characters and writing affinity rules to give appropriate social weight to an interest, two groups of characters formed.

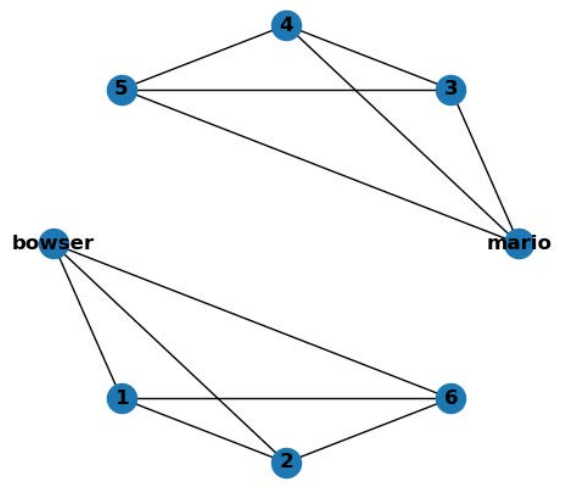

Figure 2: This network shows two distinct cliques between Bowser and Mario based on positive affinity.

If less harsh of a distinction between cliques is desired, less intense affinity rules can be used or less limiting constraints on pair affinity. An example of this can be seen in figure 3. Here rather than 2 cliques forming, more cliques form but they are now less polarized. This allows for a different narrative to be constructed. In the previous figure if a conflict between Mario and Bowser were to arise supporting characters would have a clear character to support because there are no conflicting alliances. In figure 3 there is a more nuanced network of characters that has formed.

The cast in figure 3 has only one supporting character close to Mario whereas Bowser has two close supporting characters. Characters such as character 3 have interesting degrees of separation between Bowser and Mario. Character 3 is close to neither but has a mutual person they have positive affinity towards. 


\section{NPC Personality \& Social Connection Generation}

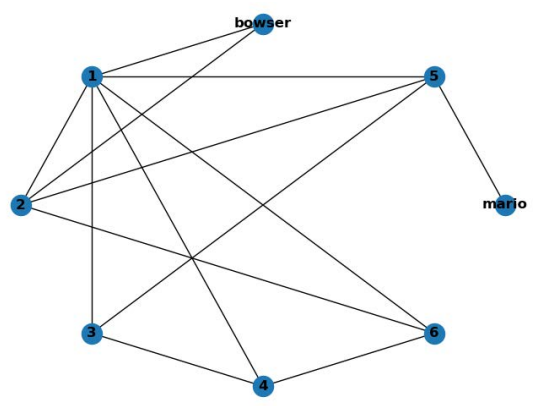

Figure 3: With less polarizing affinity rules, less distinct cliques form. Characters do not all have a clear alliance to either Bowser or Mario and have a more nuanced conflict may arise if they were forced to choose.

Aggregate incoming and outgoing affinity also may help contribute to the narrative. In the example given in the tech description and shown in figure 2, the characters have the aggregate affinity seen in figure 4 . Due to constraints focused on the negative feelings that arise from differences and similarities in the interest to kidnap princesses, aggregate incoming and outgoing affinity is overwhelmingly negative. Incoming and outgoing affinity for a given character can also vary drastically. In this solution Mario is the only character who has a slight positive incoming affinity and despite others viewing him most positively overall, he has negative affinity towards others overall. Bowser appears to be a misunderstood antagonist. Others view him poorly, but he still feels more positively about others than any other character. In contrast characters 3,4 , and 5 have intense negative feelings towards others.

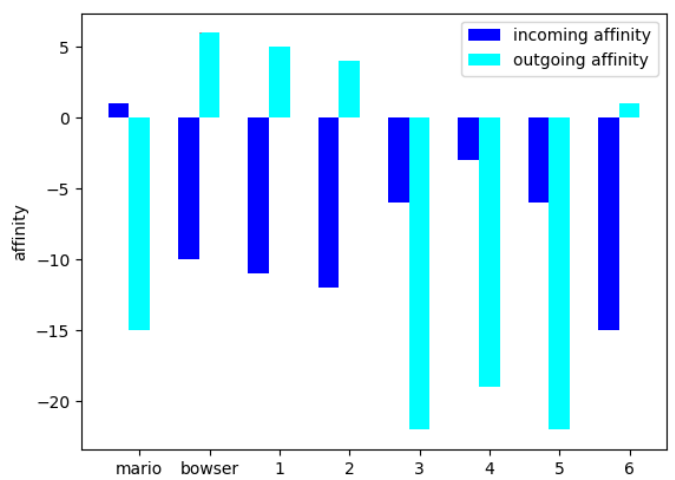

Figure 4: This plot shows the generated aggregate incoming and outgoing affinity for the cast of characters with cliques formed around Mario and Bowser as seen in figure 2. With two distinct cliques and with harsh affinity rules, strong negative feelings arise between characters.
FDG'19, August 26-30, 2019, San Luis Obispo, CA

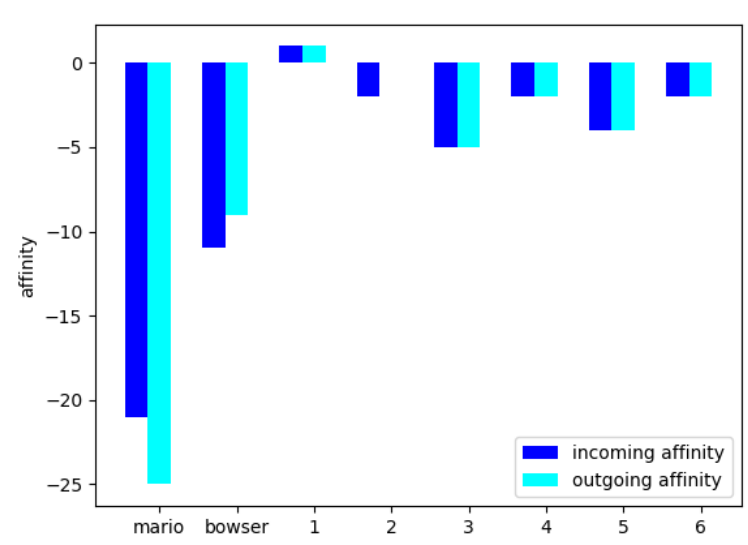

Figure 5: With less extreme affinity rule intertwined cliques form. In this system incoming and outgoing affinity for the cast of characters is less negative. The incoming and outgoing affinity for all characters are much more balanced.

In the solution with less harsh affinity rules and more cliques, the aggregate incoming and outgoing affinity is very different. As seen in figure 5 , incoming and outgoing affinity are much more similar. In contrast with the previous solution the protagonist, Mario, is more disliked than the antagonist, Bowser.

As seen in these two examples, even the same personality traits, interests, and cast size can result in different cast dynamics through similarity and affinity rules that describe the desired relationships. Through iteratively refining the design space through CAST, new solutions with different dynamics may inspire the author to modify the emergent narratives. Overall, through careful crafting of constraints, CAST can be used to generate a cast of characters whose feelings towards each other are grounded in what the is most important in the game world.

\section{Future Work}

Personality and interests are both core to who we are as people and impacts the relationships we form with others. However, identity is also composed of our needs, wants, values, and desires and the relationships we form are highly dependent on this. Deep committed relationships, whether they are familial, romantic, or platonic, are possible when individuals have compatible values and wants. Liking the same kind of craft beer may be enough to make and maintain a casual work acquaintance, but for a committed partnership to work compatibility must extend beyond interests. Long term desires concerning one's career, family, money, children, and more must align or at least not be diametrically opposed even if personality and interests click. CAST currently is unable to take this into account but would be improved greatly if this were considered.

In future iterations a cultural knowledgebase module should be added so interests and objects can be more robustly described. 


\section{NPC Personality \& Social Connection Generation}

Objects or interests should have information about how they are viewed in the game world [7]. Interests can provide a commonality for characters to bond over. Besides providing common ground, interests also are significant because they may influence the way others see them. This social significance of interests is currently somewhat lost in CAST although affinity rules can help correct this.

CAST also should have a more user-friendly interface. Rather than having the user write a few lines of Clingo code, CAST should have an intuitive graphical interface where the user can select the cast size, facets, and interests easily. Additionally, the interface should visualize the solution. This solution should be interactive such that nodes and connections can be selected and edited. This would greatly improve CAST's ability to work as an iterative design tool. Currently authors must know Clingo to make use of CAST. Specifying characters, facets, and interests is relatively simple to do but complex affinity rules may be challenging to write without a background in ASP. A GUI would remove this problem and help ensure that CAST is more accessible to users of all experience levels.

More abstract analysis of the answer set should also be provided by CAST. An answer set containing the characters, their facets, their interests, and the network of similarity and affinity values is generated. Not only should this be displayed in the interface, corresponding summary information should be generated. Although some high-level summary of the system exists because the constraints inherently describes it, more salient information should be extracted. Information about centrality, clustering, cliques, outgoing and ingoing affinity, and similar metrics should be provided to the author. This could aid in understanding the given solution and iteratively refining it to reach a desirable solution. For a small cast of characters these evaluation metrics are less essential, but for larger cast of characters these sorts of summary metrics could help evaluate the solution. In the future these high-level summaries should also be turned into constraints so CAST can accommodate requests like making a number of cliques. CAST provides a higher level of abstraction and control through constraints on affinity and similarity, but another layer of abstraction to control overall emergent properties of the cast would greatly help the author.

\section{Conclusion}

Cast Affinity Satisfiability Toolkit uses answer set programming as both a means to enforce author autonomy and as a generative tool to find socially appropriate and consistent personality facets, interests, and relationships between characters. Through allowing constraints on similarity and affinity, CAST provides a method to describe not only the traits of desired cast characters but the social network that binds them. Affinity rules, or rules that define how characters are attracted to each other, can be used to weight personality facets and interests to generate a cast of characters and relationships with the appropriate social significance.
FDG'19, August 26-30, 2019, San Luis Obispo, CA

Although the quality of output is contingent upon the quality of the constraints, CAST respects all author choices and provides the framework to generate consistent NPCs whose relationships reflect what is significant in the story world. This generative process lessens the time needed to generate large numbers of NPCs and allows for an iterative refinement process to create characters and form the narrative about them.

\section{REFERENCES}

[1] Jan M Allbeck and Norman I Badler. Creating Crowd Variation with the Ocean Personality Model. 6.

[2] Paul T. Costa Jr. and Robert R. McCrae. 1995. Domains and Facets: Hierarchical Personality Assessment Using the Revised NEO Personality Inventory. 7. Pers. Assess. 64, 1 (February 1995), 21-50. DOI:https://doi.org/10.1207/s15327752jpa6401_2

[3] Martin Gebser, Roland Kaminski, Benjamin Kaufmann, and Torsten Schaub. 2017. Multi-shot ASP solving with clingo. ArXiv170509811 Cs (May 2017). Retrieved May 3, 2019 from http://arxiv.org/abs/1705.09811

[4] Martin Gebser, Roland Kaminski, Benjamin Kaufmann, and Torsten Schaub. Clingo = ASP + Control: Extended Report. 16.

[5] Ian Horswill. Postmortem: MKULTRA, An Experimental AI-Based Game. Retrieved May 3, 2019 from https://aaai.org/ocs/index.php/AIIDE/AIIDE18/paper/view/18123

[6] Antonios Liapis, Gillian Smith, and Noor Shaker. 2016. Mixed-initiative content creation. In Procedural Content Generation in Games. Springer International Publishing, Cham, 195-214. DOI:https://doi.org/10.1007/978-3319-42716-4_11

[7] Joshua Allen McCoy. All the World's a Stage: A Playable Model of Social Interaction Inspired by Dramaturgical Analysis. 331.

[8] Mark J. Nelson and Adam M. Smith. 2016. ASP with Applications to Mazes and Levels. In Procedural Content Generation in Games. Springer International Publishing, Cham, 143-157. DOI:https://doi.org/10.1007/978-3319-42716-4_8

[9] Yanko Oliveira. 2017. A Primer on Procedural Character Generation for Games and Real-Time Applications. In Game Dynamics: Best Practices in Procedural and Dynamic Game Content Generation, Oliver Korn and Newton Lee (eds.). Springer International Publishing, Cham, 115-132. DOI:https://doi.org/10.1007/978-3-319-53088-8_7

[10] Adam M. Smith and Michael Mateas. 2011. Answer Set Programming for Procedural Content Generation: A Design Space Approach. IEEE Trans. Comput. Intell. AI Games 3, 3 (September 2011), 187-200. DOI:https://doi.org/10.1109/TCIAIG.2011.2158545

[11] 2011. Star Wars: The Old Republic. BioWare. Retrieved from https://www.swtor.com/

[12] 2013. BioShock Infinite. $2 \mathrm{~K}$ Games. Retrieved from https://2k.com/enUS/game/bioshock-infinite/

[13] 2016. Final Fantasy XV. Square Enix. Retrieved from https://finalfantasyxv.square-enix-games.com/ 